\title{
Stability Analysis of Networked Control in Smart Grids
}

\author{
Abhinav Kumar Singh, Student Member, IEEE, Ravindra Singh, Member, IEEE, and Bikash C. Pal, Fellow, IEEE
}

\begin{abstract}
A suitable networked control scheme and its stability analysis framework have been developed for controlling inherent electromechanical oscillatory dynamics observed in power systems. It is assumed that the feedback signals are obtained at locations away from the controller/actuator and transmitted over a communication network with the help of phasor measurement units (PMUs). Within the generic framework of networked control system (NCS), the evolution of power system dynamics and associated control actions through a communication network have been modeled as a hybrid system. The data delivery rate has been modeled as a stochastic process. The closed-loop stability analysis framework has considered the limiting probability of data dropout in computing the stability margin. The contribution is in quantifying allowable data-dropout limit for a specified closed loop performance. The research findings are useful in specifying the requirement of communication infrastructure and protocol for operating future smart grids.
\end{abstract}

Index Terms-Cyber physical energy network, damping control, Kalman filtering, linear quadratic Gaussian (LQG), networked control systems (NCS), packet-drop, phasor measurement units (PMUs), smart grid, stochastic stability, thyristor controlled series capacitor (TCSC).

\section{INTRODUCTION}

$\mathbf{E}$ LECTRICAL energy systems all over the world have been undergoing unprecedented level of transitions in recent times. The transitions are primarily driven by the requirement to renew the aging electricity infrastructure; to integrate low-carbon intermittent energy sources; to meet increased power consumption and new forms of demands such as electric transport, smart home and business; and to ensure the security of supply. Several enabling technologies such as power electronics, wide area measurements (WAMS) and the information and communication technology (ICT) are increasingly getting deployed in the system to deliver the requirement. Such deployments increase the complexity and challenges of the system operation and the electricity grid of the 21 st century has to embrace these challenges through planning, design, operation and control. In the operation time-scale ensuring the security and stability of the system are important tasks. The WAMS and ICT offer very effective means of delivering these objectives ([1] and [2]).

Manuscript received November 14, 2013; revised February 19, 2014 and March 24, 2014; accepted March 27, 2014. Date of publication May 09, 2014; date of current version December 17, 2014. This work was supported by EPSRC, U.K., under Grant EP/G066477/1. Paper no. TSG-00854-2013.

A. K. Singh and B. C. Pal are with the Control and Power Group, Department of Electrical and Electronic Engineering, Imperial College London, SW7 2BT, London (e-mail: a.singh11@imperial.ac.uk; b.pal@imperial.ac.uk).

R. Singh is with the ABB Corporate Research Centre, Rayleigh, NC, USA (e-mail: singh.ravindra@gmail.com).

Color versions of one or more of the figures in this paper are available online at http://ieeexplore.ieee.org.

Digital Object Identifier 10.1109/TSG.2014.2314494
The networked control system (NCS) approach utilizing modern measurements and communications is very appropriate in this context. In NCS the control loops are closed through a real-time network where the control and feedback signals are exchanged among the system's components in the form of packets of data and information [5]. It enables execution from long distance by connecting cyberspace to physical space. It has been successfully applied in other technology areas such as space and terrestrial exploration, aircraft, automobiles, factory automation and industrial process control. The Packet-switching based communication networks are the most widely adopted systems for fast, economic and stable data transfer over both large and small distances through dynamic path allocation. They are in contrast to the traditional circuit-switching based networks in which a dedicated link is established between the sending and the receiving ends. Circuit switching is not only inefficient and costlier than packet-switching, but also the link failure rate increases for large transmission distances, and the failure cannot be dynamically corrected, unlike packet-switching [3], [4]. This is the reason that most of the current research in NCS is based on packet-switching technology. The NCS offers many advantages over the traditional control architectures, including low cost of installation, ease of maintenance and greater flexibility. They also suffer from some problems such as packet-dropout, network induced delays and packet-disordering [6]. These factors can possibly degrade the performance of the control of important power system dynamics such as system wide electromechanical oscillations, more commonly known as inter-area oscillations of the system [7]. In the context of interconnected power systems, the control of oscillatory stability is very time critical as uncontrolled oscillations in the past have led to several power blackouts [8]. Therefore one needs to analyze these factors thoroughly for assessing the suitability of the NCS approach to wide area control of power systems.

Over the past decade, substantial research has been undertaken to model NCS and study the effect of packet-dropout and time delays on the control design and the stability of the NCS ([5], [6], [9] and [10]), but this research is not reflected in the power system literature. In most of the literatures relating to power systems, it is assumed that the transmission of signals to and from the central control unit occurs over an ideal, lossless and delay-free communication network. A few exceptions to this are [11], [12] and [13]. In [11] the effect of network induced time-delays has been considered using a WAMS based state-feedback control methodology. In [12] an estimation of distribution algorithm (EDA) based speed control of networked DC motor system has been studied; and in [13] the effect of communication-bandwidth constraints on the stability of 
WAMS based power system control has been studied. But all these papers have other limitations. For instance, in [11] it is not explained how the various system states (such as the rotor angle, rotor velocity and transient voltages) are estimated before using them for state-feedback; and also the power system model considered in the paper is too simplistic to represent actual power system dynamics. In [12] only a local network based control of a single dc-motor system is considered instead of considering the networked control of a complete power-system. In [13], the chief problems associated with networked-control, which are packet-loss and delay, are not considered.

This paper has made an attempt to address the aforementioned limitations by analyzing the effects of packet-dropout on the oscillatory stability response of a networked controlled smart grid (NCSG). A Kalman filtering and linear quadratic Gaussian (LQG) based optimal control scheme for damping oscillatory stability of the NCSG has been adopted in the paper. Specifically, the contributions of the paper may be summarized as follows:

- A detailed characterization of packet transmission process and the probability of packet loss have been considered in the framework of NCS for power system control.

- A practical output-feedback methodology has been used for control (instead of state-feedback), and the signals which are required to be transmitted to the control unit are measureable line-power signals. Also, a detailed and realistic sub-transient power system model has been used.

- The LQG control scheme used in the paper is an optimal control scheme for a networked system in which packet loss is considered. Moreover, the control scheme can be easily integrated with the WAMS or flexible AC transmission system (FACTS) devices already present in the system, and any extra investment is not required.

A rigorous model for a NCSG is presented in Section II. Section III presents LMI based stability analysis of the developed NCSG, and derives the probability threshold of the packet-dropout rate while guaranteeing specified level of damping of the NCSG. A case study of a representative 68-bus New-England/New-York inter-connected NCSG model has been presented in Section IV. In the case study, the inter-area oscillations in the power system are controlled using feedback signals which are transmitted over a communication network. Section V concludes the paper.

\section{NCSG Modeling With OUTPUT-FEEDBACK}

A block diagram of the output-feedback controlled NCSG is shown in Fig. 1. The model is described as a hybrid continuousdiscrete system in which the power system is the continuous, while the networked-controller is the discrete part.

In Fig. 1, the block "Power System" represents the open-loop power system whose oscillatory dynamics is to be controlled. To this effect, real-power deviations in some of the lines are measured in real-time using current transformers (CTs) and potential transformers (PTs), and represented by $\boldsymbol{y}(t)$ in the block diagram. These are then sampled at the sampling rate of the communication network using digital devices such as phasor measurement units (PMUs) and intelligent electronic devices

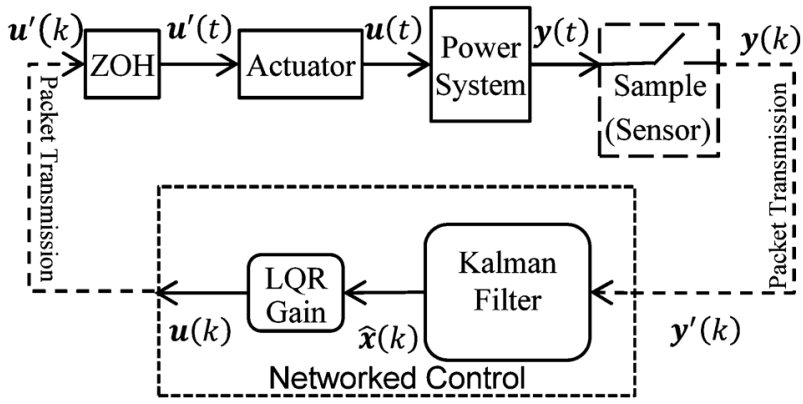

Fig. 1. A reduced model of the NCSG.

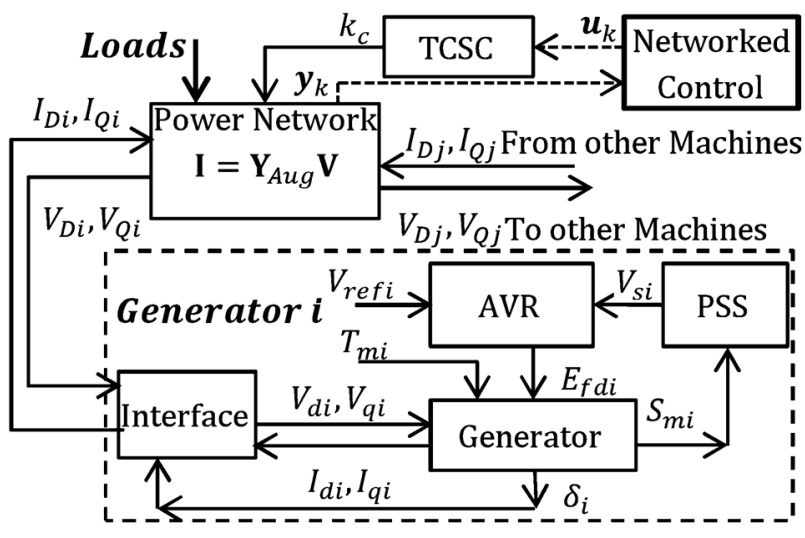

Fig. 2. Details of the power system in the NCSG.

(IEDs) and then sent over the communication network as discrete data-packets, $\boldsymbol{y}(k)$. User datagram protocol (UDP) is used for packet transmission, and packet-loss occurs during transmission. The final data which is received at the control unit after packet loss is given by $\boldsymbol{y}^{\prime}(k)$. The control unit consists of a LQG controller, which is a combination of a Kalman filter and a linear quadratic regulator (LQR). Kalman filter uses the reduced-power system model and the output data-packets arriving at the controller, $\boldsymbol{y}^{\prime}(k)$, to estimate the states, $\hat{\boldsymbol{x}}(k)$. The state estimates are then multiplied by the LQR gain to produce the control signals $\boldsymbol{u}(k)$, which are then sent over the communication network to the actuators. The packets arrive at discrete to analog converters (DACs), which are zero order hold devices and convert the discrete control signals after packet-loss, $\boldsymbol{u}^{\prime}(k)$, into continuous control signals, $\boldsymbol{u}^{\prime}(t)$. These continuous signals control the actuators, which are the FACTS devices, more commonly known as FACTS controllers. The inputs $\boldsymbol{u}(t)$ to the power system are the percentage compensations provided by the FACTS controllers to control the power-flow in the lines on which the FACTS controllers are installed. All the variables in the model have been expressed in per unit (p.u.), except the time variables which are expressed in $\mathrm{s}$. A detailed description of each component of the NCSG model is presented as follows.

\section{A. Power System}

An interconnected power system is represented through important components such as the generators, their excitation systems, power system stabilizers (PSS), FACTS controllers such as a thyristor controlled series capacitor (TCSC), loads and transmission network [15] as shown in Fig. 2. 
The dynamics of the system is modeled using a set of non-linear differential and algebraic equations (DAEs) ([14] and [15]). The state space representation of the system is obtained through linearization of the DAEs around an initial operating point. The order of the system is generally reduced to speed up the controller design algorithm. On applying a standard balanced model reduction given in [16], only the unstable and/or poorly damped electromechanical modes of the power system are retained in the reduced model. The reduced model is written as:

$$
\begin{aligned}
\Delta \dot{\boldsymbol{x}}(t) & =\boldsymbol{A}_{R} \Delta \boldsymbol{x}(t)+\boldsymbol{B}_{R} \Delta \boldsymbol{u}(t) \\
\Delta \boldsymbol{y}(t) & =\boldsymbol{C}_{R} \Delta \boldsymbol{x}(t)
\end{aligned}
$$

$\boldsymbol{A}_{R} \in \mathbb{R}^{n \times n}, \boldsymbol{B}_{R} \in \mathbb{R}^{n \times p}$ and $\boldsymbol{C}_{R} \in \mathbb{R}^{q \times n}$ are the reduced state space matrices and $\boldsymbol{x} \in \mathbb{R}^{n}, \boldsymbol{u} \in \mathbb{R}^{p}$ and $\boldsymbol{y} \in \mathbb{R}^{q}$ are the vectors of reduced-state variables, inputs and outputs, respectively. It should be noted that after balanced reduction of the full model, only the state variables and the state matrices get reduced in order; the inputs $\boldsymbol{u}$ and the outputs $\boldsymbol{y}$ remain same as in the original full model. Also, out of the various possible measurable outputs (which are the line-powers in the context of NCSG), only those outputs are selected in $\boldsymbol{y}$ which have high observability of the unstable and/or poorly damped electromechanical modes of the power system.

\section{B. Sensors and Actuators}

The sensors (in the context of NCSG, they are CTs, PTs and PMUs) send the feedback signals to the controller over the communication network at a regular interval of $T_{s}$, which is the sampling period of the communication network. The discrete to analog converters (DACs) convert the discrete control signals after packet-loss into continuous control signals. The DACs are event-driven zero-order-hold ( $\mathrm{ZOH})$ devices, each one of which holds the input to the power system in a given cycle. In the next cycle it holds its previous value if there is no new input due to packet drop, otherwise it holds the new input. The outputs of the DACs control the FACTS controllers, which are the actuators; and the inputs $\boldsymbol{u}(t)$ to the power system are the percentage compensations provided by the FACTS controllers. For the $(k+1)$ th time cycle, (1) reduces to:

$$
\Delta \dot{\boldsymbol{x}}(t)=\boldsymbol{A}_{R} \Delta \boldsymbol{x}(t)+\boldsymbol{B}_{R} \Delta \boldsymbol{u}\left(k T_{s}\right) ; 0 \leq t-k T_{s}<T_{s}
$$

Solving (3) with initial condition $\left(\Delta \boldsymbol{x}\left(k T_{s}\right), \Delta \boldsymbol{u}\left(k T_{s}\right)\right)$ and a constant input $\Delta \boldsymbol{u}\left(k T_{s}\right)$ [17], we get:

$$
\begin{aligned}
\Delta \boldsymbol{x}\left((k+1) T_{s}\right)= & \boldsymbol{A} \Delta \boldsymbol{x}\left(k T_{s}\right)+\boldsymbol{B} \Delta \boldsymbol{u}\left(k T_{s}\right) ; \\
& \boldsymbol{A}=e^{\boldsymbol{A}_{R} T_{s}} ; \boldsymbol{B}=\boldsymbol{A}_{R}^{-1}\left(e^{\boldsymbol{A}_{R} T_{s}}-\boldsymbol{I}\right) \boldsymbol{B}_{R}
\end{aligned}
$$

Denoting $\Delta \boldsymbol{x}\left(k T_{s}\right)$ as $\boldsymbol{x}_{k}, \Delta \boldsymbol{u}\left(k T_{s}\right)$ as $\left(\boldsymbol{u}_{k}^{\prime}+\boldsymbol{w}_{k}\right)$ (where $\boldsymbol{u}_{k}^{\prime}$ is the uncertain input after packet dropout while $\boldsymbol{w}_{k}$ is the white Gaussian noise at actuators), $\Delta \boldsymbol{y}\left(k T_{s}\right)$ as $\boldsymbol{y}_{k}, \boldsymbol{C}_{R}$ as $\boldsymbol{C}$, and the white Gaussian noise at the sensors as $\boldsymbol{v}_{k}$, we get:

$$
\boldsymbol{x}_{k+1}=\boldsymbol{A} \boldsymbol{x}_{k}+\boldsymbol{B} \boldsymbol{u}_{k}^{\prime}+\boldsymbol{B} \boldsymbol{w}_{k} ; \boldsymbol{y}_{k}=\boldsymbol{C} \boldsymbol{x}_{k}+\boldsymbol{v}_{k}
$$

\section{Communication Protocols and Packet Delays and Dropouts}

In the model design process two classes of communication protocols have been considered. In transmission control pro-

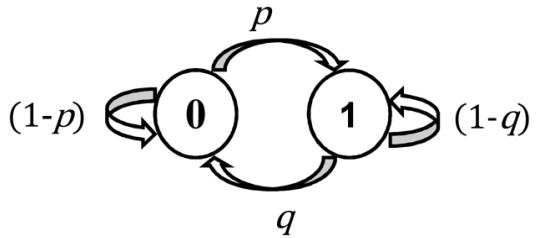

Fig. 3. Markov chain for Gilbert process.

tocol (TCP)-like protocols the acknowledgments that the receiver received the packets are sent back to the sender, while in user datagram protocol (UDP)-like protocols they are not sent. In TCP-like case, unlike in the UDP-like case, the lost packets can be re-sent because of the availability of the acknowledgments. So the separation principle, as explained in [18], holds only in the case of TCP-like protocols, and hence the controller and the estimator can be designed independently [19]. In UDP-like case no known optimal regulator exists and one can design a suboptimal solution based on a Kalman-like estimator and a LQG-like state feedback controller, as shown in Fig. 1. Although UDP-like protocol results in a sub-optimal solution, it is preferred over a TCP-like protocol as it may be extremely difficult to both analyze and implement a TCP-like control scheme [19]. In this paper, a UDP-like scheme has been used. The time delays and dropouts of packets have been modeled such that a packet is assumed to be lost, unless its time-delay is less than the sampling interval of the system. This fact is one of the factors while deciding the sampling duration, the other factor being the type of control needed, as explained in Section IV-B1. If a packet is lost, the output of the receiver is held at the last successfully received packet.

The packet loss over the network usually follows a random process. In the present analysis an independent Bernoulli process has been used to model the packet loss [19]. The input $\boldsymbol{u}_{k}^{\prime}$ at the actuator and $\boldsymbol{y}_{k}^{\prime}$ at the estimator are modeled as:

$$
\boldsymbol{u}_{k}^{\prime}=\boldsymbol{\alpha}_{k} \boldsymbol{u}_{k} ; \quad \boldsymbol{y}_{k}^{\prime}=\boldsymbol{\beta}_{k} \boldsymbol{y}_{k}
$$

$\boldsymbol{\alpha}_{k}=\operatorname{diag}\left(\alpha_{k}^{1}, \alpha_{k}^{2}, \ldots, \alpha_{k}^{p}\right)$ is a stationary diagonal binary random matrix, in which the value of $\alpha_{k}^{i}$ is equal to one with a probability $p_{u i}$, indicating that the $i$ th component of $\boldsymbol{u}_{k}$ is delivered; while its value is equal to zero with a probability $\left(1-p_{u i}\right)$, indicating that the component is lost.

Remark: The assumption of an independent Bernoulli packet loss model is not valid when the communication channel is congested. In a congested channel the packet loss occurs in bursts, and follows a two-state Markov chain model, also known as Gilbert model [20]. Fig. 3 shows this model, where "1" represents the state of packet delivery and " 0 " represents the state of packet loss; and the probability of transition from state " 0 " to state " 1 " is $p$ and the probability of transition from state " 1 " to state " 0 " is $q$. When $p$ is equal to $(1-q)$, then this model reduces to Bernoulli model.

The drawback of using Gilbert model in stability analysis is that this model is not a memory-less model, which means that the probability of packet delivery depends on the current channel state, and it fluctuates between $p$ and $(1-q)$ depending on whether the current channel state is " 0 " or " 1 ," respectively. Mathematical representation of such a fluctuating probability of packet delivery becomes practically infeasible. A practical alter- 
native for approximating Gilbert model with Bernoulli model can be to set the communication channel's probability of packet delivery as $p$ if $p<(1-q)$, and as $(1-q)$ if $(1-q) \leq p$. Thus, the approximated Bernoulli model represents the worst case scenario of packet delivery performance given by Gilbert model, as the smaller probability of the two possible packet delivery probabilities from Gilbert model is assumed to be the constant packet delivery probability in the approximated Bernoulli model.

\section{Controller}

For an open-loop LTI system given by (5), whose input $\boldsymbol{u}_{k}^{\prime}$ is defined by (6), the quadratic cost function $\boldsymbol{J}$ is given by:

$$
\boldsymbol{J}=\frac{1}{N} \mathbb{E}\left\{\boldsymbol{x}_{N}^{T} \boldsymbol{Q} \boldsymbol{x}_{N}+\sum_{k=0}^{N-1}\left[\boldsymbol{x}_{k}^{T} \boldsymbol{Q} \boldsymbol{x}_{k}+\boldsymbol{u}_{k}^{T} \boldsymbol{\alpha}_{k} \boldsymbol{R} \boldsymbol{\alpha}_{\boldsymbol{k}} \boldsymbol{u}_{k}\right]\right\}
$$

where $N$ is the number of samples, $\mathbb{E}$ is the expectation value, $T$ denotes the transpose of a vector or a matrix, $\boldsymbol{Q}$ is a positive definite matrix, $\boldsymbol{R}$ is a positive semi-definite matrix and it is assumed that the full state information of the LTI system is available (we get this information from the state-estimator). Minimizing $\boldsymbol{J}$ with respect to $\boldsymbol{u}_{k}$ results in the following forward Riccati-like difference equation, as exlained in [21]:

$$
\begin{aligned}
& \boldsymbol{K}_{k+1}=\boldsymbol{A}^{T} \boldsymbol{K}_{k} \boldsymbol{A}+\boldsymbol{Q} \\
& \quad-\boldsymbol{A}^{T} \boldsymbol{K}_{k} \boldsymbol{B} \mathbb{E}[\boldsymbol{\alpha}]\left(R+\mathbb{E}\left[\boldsymbol{\alpha} \boldsymbol{B}^{T} \boldsymbol{K}_{k} \boldsymbol{B} \boldsymbol{\alpha}\right]\right)^{-1} \mathbb{E}[\boldsymbol{\alpha}] \boldsymbol{B}^{T} \boldsymbol{K}_{k} \boldsymbol{A}
\end{aligned}
$$

where $\boldsymbol{K}_{0}$ is a positive definite matrix, say an identity matrix, of the same order as $\boldsymbol{A}$ and $\mathbb{E}[\boldsymbol{\alpha}]$ is the expectation value of $\boldsymbol{\alpha}_{k}$ (subscript $k$ is removed in $\mathbb{E}[\boldsymbol{\alpha}]$ as $\boldsymbol{\alpha}_{k}$ is stationary). If we obtain a steady state solution $\boldsymbol{K}=\boldsymbol{K}_{\infty}$ for (8) as $k \rightarrow \infty$ then the LTI open-loop system is stabilizable in mean-square sense, provided the pair $(\boldsymbol{A}, \boldsymbol{B})$ is controllable; the pair $\left(\boldsymbol{A}, \boldsymbol{Q}^{1 / 2}\right)$ is observable, where $\boldsymbol{Q}=\left(\boldsymbol{Q}^{1 / 2}\right)^{T} \boldsymbol{Q}^{1 / 2}$, and perfect state information is received at every sample. The infinite horizon control policy for such a system is a state feedback policy, given by:

$$
\boldsymbol{u}_{k}=\boldsymbol{L} \widehat{\boldsymbol{x}}_{k} ; \boldsymbol{L}=-\left(R+\mathbb{E}\left[\boldsymbol{\alpha} \boldsymbol{B}^{T} \boldsymbol{K} \boldsymbol{B} \boldsymbol{\alpha}\right]\right)^{-1} \mathbb{E}[\boldsymbol{\alpha}] \boldsymbol{B}^{T} \boldsymbol{K} \boldsymbol{A}
$$

where $\widehat{\boldsymbol{x}}_{k}$ is the estimated state, and $\boldsymbol{L}$ is the LQG gain.

\section{E. Estimator}

The controller uses the output from the state estimator to generate the control command which is sent over the network to the actuator in the power system. The estimator uses the information vector, which consists of the control command and the intermittent plant output delivered to the estimator via the network, to generate a best estimate of the state of the system. It was shown in [22] that even in the case of intermittent observations, Kalman filter is still the best linear estimator for LTI systems with stationary Gaussian noise processes, provided that only time update is performed when a measurement packet is dropped. When a measurement is received, both the time and measurement update steps are performed. The filtering equations for such a closed-loop system, using (9), are:

1) Prediction step:

$$
\widehat{\boldsymbol{x}}_{k \mid k-1}=\boldsymbol{A}^{\prime} \widehat{\boldsymbol{x}}_{k-1 \mid k-1} ; \boldsymbol{A}^{\prime}=(\boldsymbol{A}+\boldsymbol{B} \mathbb{E}[\boldsymbol{\alpha}] \boldsymbol{L})
$$

$$
\widehat{\boldsymbol{P}}_{k \mid k-1}=\boldsymbol{A}^{\prime} \widehat{\boldsymbol{P}}_{k-1 \mid k-1} \boldsymbol{A}^{\prime T}+\boldsymbol{M}_{k}
$$

\section{2) Estimation step:}

$$
\begin{aligned}
\widehat{\boldsymbol{x}}_{k \mid k} & =\widehat{\boldsymbol{x}}_{k \mid k-1}+\boldsymbol{\kappa}_{k} \boldsymbol{\beta}_{k}\left(\boldsymbol{y}_{k}-\boldsymbol{C} \widehat{\boldsymbol{x}}_{k \mid k-1}\right) \\
\widehat{\boldsymbol{P}}_{k \mid k} & =\widehat{\boldsymbol{P}}_{k \mid k-1}-\boldsymbol{\kappa}_{k} \boldsymbol{\beta}_{k} \boldsymbol{C} \widehat{\boldsymbol{P}}_{k \mid k-1} \\
\boldsymbol{\kappa}_{k} & =\widehat{\boldsymbol{P}}_{k \mid k-1} \boldsymbol{C}^{T}\left[\boldsymbol{C} \widehat{\boldsymbol{P}}_{k \mid k-1} \boldsymbol{C}^{T}+\boldsymbol{B} \boldsymbol{N}_{k} \boldsymbol{B}^{T}\right]^{-1}
\end{aligned}
$$

For the kth sample, $\widehat{\boldsymbol{x}}_{k \mid k-1}$ is the predicted state, $\widehat{\boldsymbol{P}}_{k \mid k-1}$ is the predicted-state-error covariance matrix, $\widehat{\boldsymbol{x}}_{k \mid k}$ is the estimated state, $\widehat{\boldsymbol{P}}_{k^{\prime} k}$ is the estimated-state-error covariance matrix, $\boldsymbol{N}_{k}$ is the covariance matrix of $\boldsymbol{w}_{k}, \boldsymbol{M}_{k}$ is the covariance matrix of $\boldsymbol{v}_{k}, \boldsymbol{\kappa}_{k}$ is the Kalman gain. The equations are valid iff $(\boldsymbol{A}, \boldsymbol{C})$ is observable and $\left(\boldsymbol{A}, \boldsymbol{M}_{k}^{1 / 2}\right)$ is controllable. In (10) the estimator takes the closed loop state-space matrix $\boldsymbol{A}^{\prime}$ as $(\boldsymbol{A}+\boldsymbol{B} \mathbb{E}[\boldsymbol{\alpha}] \boldsymbol{L})$ as it can at best have an estimate of the packet dropout rate of the network because it does not receive the acknowledgments of the control packets it sends out to the power system.

\section{Closed-Loop Stability AND DAMPING ResPonse}

The closed loop model of the NCSG can be summarized as follows, using (5)-(14) and rewriting $\widehat{\boldsymbol{x}}_{k \mid k}$ as $\widehat{\boldsymbol{x}}_{k}$ :

$$
\begin{aligned}
& \boldsymbol{x}_{k+1}=\boldsymbol{A} \boldsymbol{x}_{k}+\boldsymbol{B} \boldsymbol{\alpha}_{k} \boldsymbol{L} \widehat{\boldsymbol{x}}_{k}+\boldsymbol{B} \boldsymbol{w}_{k} ; \\
& \widehat{\boldsymbol{x}}_{k+1}=\boldsymbol{A}^{\prime} \widehat{\boldsymbol{x}}_{k}+\boldsymbol{\kappa}_{k+1} \boldsymbol{\beta}_{k+1}\left(\boldsymbol{y}_{k+1}-\boldsymbol{C} \boldsymbol{A}^{\prime} \widehat{\boldsymbol{x}}_{k}\right) \\
& \boldsymbol{y}_{k+1}=\boldsymbol{C}\left(\boldsymbol{A} \boldsymbol{x}_{k}+\boldsymbol{B} \boldsymbol{\alpha}_{k} \boldsymbol{L} \widehat{\boldsymbol{x}}_{k}+\boldsymbol{B} \boldsymbol{w}_{k}\right)+\boldsymbol{v}_{k+1}
\end{aligned}
$$

A steady state solution for $\widehat{\boldsymbol{P}}_{k \mid k}$ in (13), and hence for $\boldsymbol{\kappa}_{k}$, may or may not exist for given $\boldsymbol{\alpha}_{k}$ and $\boldsymbol{\beta}_{k}$, even if the conditions for the existence of steady state solution for a standard Kalman filter hold; but a steady state estimate $\boldsymbol{\kappa}=\mathbb{E}\left[\boldsymbol{\kappa}_{\infty}\right]$ for the Kalman gain may be obtained by iteratively solving (11), (13) and (14) after substituting $\boldsymbol{\beta}_{k}$ with its expected value $\mathbb{E}[\boldsymbol{\beta}]$. This is the suboptimal Kalman gain which is used for deriving the condition for mean square stability and adequate damping of the developed NCSG. Writing (15)-(17) in composite form, after replacing $\boldsymbol{\kappa}_{k+1}$ with its steady state estimate $\boldsymbol{\kappa}$, we get:

$$
\begin{aligned}
& {\left[\begin{array}{l}
\boldsymbol{x}_{k+1} \\
\widehat{\boldsymbol{x}}_{k+1}
\end{array}\right]=\left[\begin{array}{c}
\boldsymbol{B} \\
\boldsymbol{\kappa} \boldsymbol{\beta}_{k+1} \boldsymbol{C}
\end{array}\right] \boldsymbol{w}_{k}+\left[\begin{array}{c}
\mathbf{0} \\
\boldsymbol{\kappa} \boldsymbol{\beta}_{k+1}
\end{array}\right] \boldsymbol{v}_{k+1}} \\
& +\underbrace{\left[\begin{array}{cc}
\boldsymbol{A} \\
\boldsymbol{\kappa} \boldsymbol{\beta}_{k+1} \boldsymbol{C A} & \boldsymbol{A}^{\prime}+\boldsymbol{\kappa} \boldsymbol{\beta}_{k+1} \boldsymbol{B}\left(\boldsymbol{B} \boldsymbol{\alpha}_{k} \boldsymbol{L}-\boldsymbol{A}^{\prime}\right)
\end{array}\right]}_{\boldsymbol{A}\left(\boldsymbol{\alpha}_{k}, \boldsymbol{\beta}_{k+1}\right)}\left[\begin{array}{l}
\boldsymbol{x}_{k} \\
\widehat{\boldsymbol{x}}_{k}
\end{array}\right]
\end{aligned}
$$

The presence of $\alpha_{k}$ and $\beta_{k+1}$ in (18) makes it a jump linear system (JLS): a system whose state matrices vary randomly with $\alpha_{k}$ and $\beta_{k+1}$. The framework of a JLS and its stability analysis are described in [23], [24] and [25]. A brief overview of the criterion for the stability and the damping in mean square sense of the NCSG has been presented in the next section.

\section{A. Stability Analysis Framework of a Jump Linear System}

Let $\boldsymbol{S}_{i}$ be a set of all the subsets of $\{1,2,3, \ldots, i\}$. Let $\boldsymbol{r} \in \boldsymbol{S}_{\boldsymbol{p}}$ be a set of indices of all those input delivery indicators whose values are one, i.e., $\boldsymbol{r}=\left\{i\right.$, such that (s.t.) $\left.\alpha_{k}^{i}=1\right\}$. E.g., for a 2 input system $(\mathrm{p}=2), \boldsymbol{r}$ can either be $\emptyset$ (both the inputs failed to deliver), or $\{1\}$ (only 1 st input delivered), or $\{2\}$ (only 2 nd 
input delivered), or $\{1,2\}$ (both the inputs delivered). Similarly, let $\boldsymbol{s} \in \boldsymbol{S}_{\boldsymbol{q}}$ be a set of indices of successful output delivery indicators. As each input delivery indicator $\alpha_{k}^{i}$ has two modes (0 or 1$)$ and $\alpha_{k}^{i} s$ are $p$ in total, $\boldsymbol{\alpha}_{k}$ has $2^{p}$ modes. Any mode of $\boldsymbol{\alpha}_{k}$ is expressed as $\boldsymbol{T}_{p}(\boldsymbol{r}), \boldsymbol{r} \in \boldsymbol{S}_{\boldsymbol{p}}$, where $\boldsymbol{T}_{p}(\boldsymbol{r})$ is a $p \times p$ diagonal matrix whose $(i, i)$ th element is 1 if $i \in \boldsymbol{r}$, else it is 0 . Similarly, $\boldsymbol{\beta}_{k+1}$ has $2^{q}$ modes, and any mode is expressed as $\boldsymbol{T}_{q}(\boldsymbol{s}), \boldsymbol{s} \in \boldsymbol{S}_{\boldsymbol{q}}$, where $\boldsymbol{T}_{q}(\boldsymbol{s})$ is a $q \times q$ diagonal matrix whose $(i, i)$ th element is 1 if $i \in \boldsymbol{s}$, else it is 0 . The probability distributions of $\boldsymbol{T}_{p}(\boldsymbol{r}), \boldsymbol{r} \in \boldsymbol{S}_{\boldsymbol{p}}$ and $\boldsymbol{T}_{q}(\boldsymbol{s}), \boldsymbol{s} \in \boldsymbol{S}_{\boldsymbol{q}}$ are given by:

$$
\begin{aligned}
& \mathcal{P}_{p}(\boldsymbol{r})=\mathbf{P}\left[\boldsymbol{\alpha}_{k}=\boldsymbol{T}_{p}(\boldsymbol{r})\right]=\prod_{i \in r} p_{u i} \prod_{i \notin r} 1-p_{u i} \\
& \mathcal{P}_{q}(\boldsymbol{s})=\mathbf{P}\left[\boldsymbol{\beta}_{k+1}=\boldsymbol{T}_{q}(\boldsymbol{s})\right]=\prod_{i \in s} p_{y i} \prod_{i \notin s} 1-p_{y i}
\end{aligned}
$$

As $A\left(\boldsymbol{\alpha}_{k}, \boldsymbol{\beta}_{k+1}\right)$ in (18) is a function of $\boldsymbol{\alpha}_{k}$ and $\boldsymbol{\beta}_{k+1}$, it may be re-expressed as $\mathcal{A}(\boldsymbol{r}, \boldsymbol{s})$ in (21):

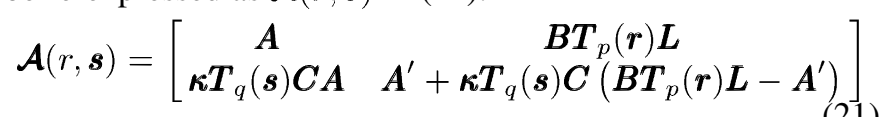

As the value of $\mathcal{A}(\boldsymbol{r}, \boldsymbol{s})$ depends on the values of $\boldsymbol{r}$ and $\boldsymbol{s}$, it can take any value in a given sample out of the possible $2^{p+q}$ values, with a corresponding overall probability distribution $\mathcal{P}_{p}(\boldsymbol{r}) \mathcal{P}_{q}(\boldsymbol{s})$. The NCSG in (18) is said to be the mean-square stable if $\lim _{k \rightarrow \infty} \mathbb{E}\left\|\begin{array}{l}\boldsymbol{x}_{k} \\ \widehat{\boldsymbol{x}}_{k}\end{array}\right\|^{2}=0$, starting with any state $\left[\begin{array}{l}\boldsymbol{x}_{0} \\ \widehat{\boldsymbol{x}}_{0}\end{array}\right]$. The mean-square stability of (18) is checked using the following methods:

1) Linear Matrix Inequalities: The criterion for the stability of discrete-time JLS in [24] is applied to obtain the condition for the mean-square stability of the NCSG in (21). The satisfaction of the criterion requires the existence of positive definite matrices $\boldsymbol{P}_{\boldsymbol{r}, \boldsymbol{s}}, \forall \boldsymbol{r} \in \boldsymbol{S}_{\boldsymbol{p}}, \forall \boldsymbol{s} \in \boldsymbol{S}_{\boldsymbol{q}}$, such that:

$$
\boldsymbol{P}_{\boldsymbol{r}, \boldsymbol{s}}>\mathcal{A}(\boldsymbol{r}, \boldsymbol{s})\left(\mathcal{P}_{p}(\boldsymbol{r}) \mathcal{P}_{q}(\boldsymbol{s}) \sum_{\boldsymbol{r}^{\prime} \in \boldsymbol{S}_{\boldsymbol{p}}, \boldsymbol{s}^{\prime} \in \boldsymbol{S}_{\boldsymbol{q}}} \boldsymbol{P}_{\boldsymbol{r}^{\prime}, \boldsymbol{s}^{\prime}}\right) \mathcal{A}(\boldsymbol{r}, \boldsymbol{s})^{T}
$$

There are in total $2^{p+q}$ LMIs in (22) and hence this method may be practically used only when the inputs and the outputs to the system are small in number. For a system with relatively larger number of inputs and outputs, the following stochastic stability analysis for JLS has been used.

\section{2) Stochastic Eigenvalue Analysis:}

Lemma 1: Consider the closed-loop NCSG described by (18). For given packet delivery probabilities (PDPs) $\boldsymbol{P}_{\boldsymbol{u}}=$ $\operatorname{diag}\left(p_{u 1}, p_{u 2}, \ldots, p_{u p}\right)$ and $\boldsymbol{P}_{\boldsymbol{y}}=\operatorname{diag}\left(p_{y 1}, p_{y 2}, \ldots, p_{y q}\right)$, the NCSG is mean-square stable iff $\boldsymbol{M}\left(\boldsymbol{P}_{\boldsymbol{u}}, \boldsymbol{P}_{\boldsymbol{y}}\right) \in \mathbb{R}^{4 n^{2} \times 4 n^{2}}$, is Schur stable (all of its eigenvalues are within unity circle).

Various elements of $\boldsymbol{M}\left(\boldsymbol{P}_{\boldsymbol{u}}, \boldsymbol{P}_{\boldsymbol{y}}\right)$ are defined, explained and followed by the proof of this lemma in Appendix A.

\section{B. Analysis of Damping Response}

The concept of $\mathcal{D}$-stability [26] has been used to study the adequate damping response of the developed NCSG. This is very practical and useful in the context of power oscillation damping. If $\mathcal{D}$ is a sub-region of the complex left half plane, and all the closed loop poles of a dynamical system $\dot{\boldsymbol{x}}=\boldsymbol{A x}$ lie in $\mathcal{D}$, then the system and its state transition matrix $\boldsymbol{A}$ are called $\mathcal{D}$-stable.

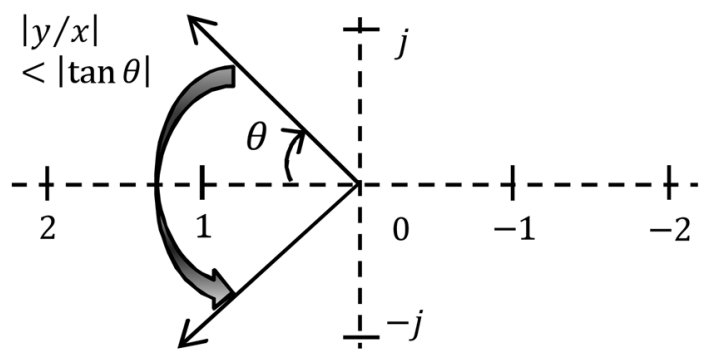

Fig. 4. $\mathcal{D}$-stability region for damping control of a continuous system.

When $\mathcal{D}$ is the entire left-half plane, then $\mathcal{D}$-stability reduces to asymptotic stability. For damping control analysis, the $\mathcal{D}$-region of interest is $\mathcal{D}(\theta)$ of complex numbers $(x+j y)$ s.t. $|y / x|<$ $|\tan \theta|$ (Fig. 4). Thus a specified and required damping of interarea modes becomes an important criterion for NCS design and analysis. Confining the closed loop poles of the system to this region ensures a minimum damping ratio $\zeta_{0}=\cos \theta$. This in turn bounds the decay rate and the settling time for the corresponding oscillatory inter-area modes of the system. Power systems usually require an operating constraint that all the disturbances in the system should settle to less than a fixed percent (usually 15\%) of the maximum overshoot within a few seconds (usually 10-15 s) of the start of the disturbance to the system [28]. As the inter-area modes usually lie in the frequency range $0.2-1.0 \mathrm{~Hz}$, they have longer settling times and lower decay rates than other modes. In this paper, the closed-loop inter-area modes have been considered to be damped to more than ten percent.

Lemma 2: The closed-loop NCSG in (18) is expected to have all of its equivalent continuous-time poles with damping ratios $\zeta>\cos \theta$ iff there exist positive definite matrices $\boldsymbol{Q}_{\boldsymbol{r}, \boldsymbol{s}}, \forall \boldsymbol{r} \in$ $\boldsymbol{S}_{\boldsymbol{p}}, \forall \boldsymbol{s} \in \boldsymbol{S}_{q}$, such that:

$$
\begin{aligned}
(\boldsymbol{W} & \left.\otimes \mathcal{A}_{\boldsymbol{c}}(\boldsymbol{r}, \boldsymbol{s})\right) \boldsymbol{Q}_{\boldsymbol{r}, \boldsymbol{s}}+\boldsymbol{Q}_{\boldsymbol{r}, \boldsymbol{s}}\left(\boldsymbol{W} \otimes \mathcal{A}_{\boldsymbol{c}}(\boldsymbol{r}, \boldsymbol{s})\right)^{T} \\
& +\left(\mathcal{P}_{p}(\boldsymbol{r}) \mathcal{P}_{q}(\boldsymbol{s})-1\right) \boldsymbol{Q}_{\boldsymbol{r}, \boldsymbol{s}} \\
& +\mathcal{P}_{p}(\boldsymbol{r}) \mathcal{P}_{q}(\boldsymbol{s})\left(\sum_{\boldsymbol{r}^{\prime} \in \boldsymbol{S}_{\boldsymbol{p}}, \boldsymbol{s}^{\prime} \in \boldsymbol{S}_{\boldsymbol{q}}, \boldsymbol{r}^{\prime} \neq \boldsymbol{r}, \boldsymbol{s}^{\prime} \neq \boldsymbol{s}} \boldsymbol{Q}_{\boldsymbol{r}^{\prime}, \boldsymbol{s}^{\prime}}\right)<0
\end{aligned}
$$

where, $\mathcal{A}_{\boldsymbol{c}}(\boldsymbol{r}, \boldsymbol{s})=\mathfrak{l n}(\boldsymbol{A}(\boldsymbol{r}, \boldsymbol{s})) / T_{s}, W=\left[\begin{array}{cc}\sin \theta & \cos \theta \\ -\cos \theta & \sin \theta\end{array}\right]$, $\mathfrak{l n}$ is the natural logarithm of a square matrix and $T_{s}$ is the sampling-period of the NCSG.

Appendix B has the proof of this lemma.

Remark: If all the channels have same characteristics, then their PDPs become equal to each other $\left(p_{u i}=p_{y i}=p_{y 0} \forall i\right)$. The marginal packet delivery probability (MPDP), such that the NCSG remains properly damped $\forall p_{y 0}>$ MPDP, is given by $\sup \left\{\gamma>0\right.$, s.t. LMIs in (23) remain feasible, $\left.\forall p_{y 0} \in[\gamma, 1]\right\}$.

\section{Physical Significance of Lemma 1 and Lemma 2 in the Context of Power Systems}

The physical meaning of the mathematical results given by Lemma 1 and Lemma 2 will be better understood using the concepts of observability and controllability. As mentioned in Section II-A, the output measurements have high observability of the unstable and/or poorly damped electromechanical modes of the power system. The LQG controller requires the knowledge of these measurements and the state matrices in order to 
correctly estimate the states, which are then multiplied by the LQR gain to get the control input for the power system. The LQG controller requires the knowledge of these measurements and the state matrices in order to correctly estimate the states, which are then multiplied with the LQR gain to get the control input for the power system. The closed loop system is properly stabilized and damped, provided the packet delivery rate is $100 \%$. The decrease in packet delivery rate from $100 \%$ results in the loss of the output measurements in the communication network. The measurements which finally arrive at the controller after packet loss have an overall decrease in their observability for a given period of time, and the controller estimates the states with decreased accuracy. For a packet delivery rate of zero percent, none of the measurements arrive at the LQG controller, and thus the observability is zero, and controller cannot estimate the states at all.

This concept of probabilistic observability will be better understood with an example. For example, if there are two measurements which are sent over the network then there are four possibilities in a given time sample: 1) none of the measurements arrive, 2) only first measurement arrives, 3) only second measurement arrives, and 4) both the measurements arrive. Each of these four possibilities has a probability associated with it depending on the packet delivery rates of the two communication channels. The overall observability of the arriving measurements depends on these four probabilities, and is thus a probabilistic quantity in itself. For $q$ measurements, there are $2^{q}$ probabilities, and the overall observability will depend on all of these probabilities. Similar analogy applies for the controllability of the power system by the control inputs sent over the communication network, and thus the overall controllability is also a probabilistic quantity. The stability and the $\mathcal{D}$-stability of the closed loop system depend on these probabilistic observability and controllability, and are written in mathematical forms as Lemma 1 and Lemma 2, respectively.

\section{CASE Study: 16-Machine 5-AREa NCSG}

\section{A. System Description}

A 16-machine, 68 bus model test system, shown in Fig. 5, has been used for the case study. This is a reduced order equivalent of the interconnected New England test system (NETS) and New York power system (NYPS) of the 1970s. NETS and NYPS are represented by a group of generators, while the power import from each of the three other neighboring areas are approximated by equivalent generator models (G14 to G16). NYPS needed to import around $1.5 \mathrm{GW}$ from Area 5, for which a TCSC was installed on the 18-50 tie-line. It was needed to dynamically control the percentage compensation of the TCSC to control the reactance of the tie-line. A detailed system description is available in [28] which is used to simulate in MATLAB SIMULINK.

\section{B. Simulation Results and Discussion}

1) Operating Condition 1 (Base Case): Total Tieline Flow Between NETS and NYPS $=700 \mathrm{MW}$, No Line Outages: For the first case of system operation, the damping and the frequency of the three poorly damped modes of the linearized system were computed. The result is displayed in Table I. The

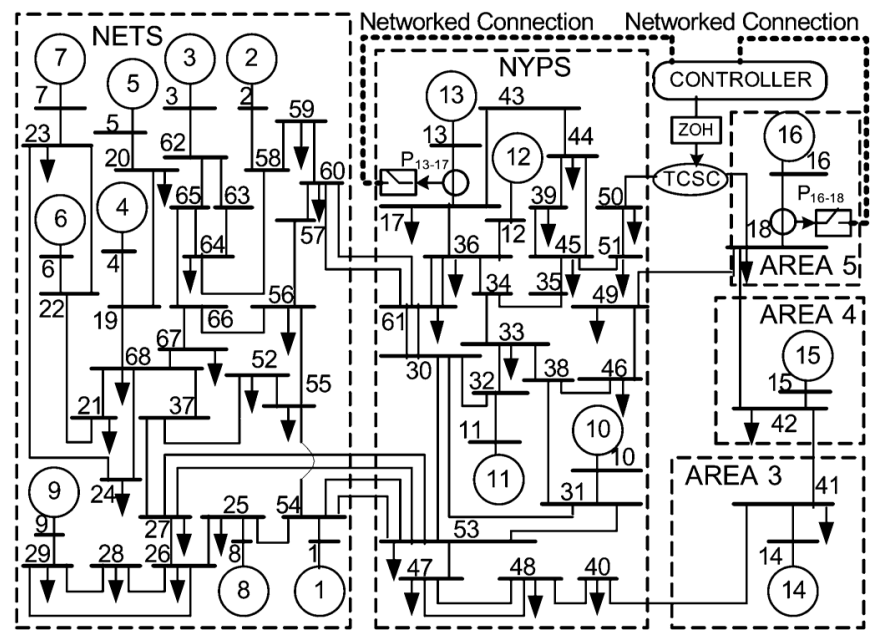

Fig. 5. Line diagram of the 16-machine, 68-bus, 5-area NCSG.

TABLE I

Normalized Residues of the ACtive Power-Flows IN tHe 3 Modes

\begin{tabular}{|c|c|c|c|c|c|}
\hline \multicolumn{2}{|l|}{ Mode $1, \zeta=0.020}$, & \multicolumn{2}{|c|}{ Mode $2, \zeta=0.041}$, & \multicolumn{2}{c|}{ Mode 3, $\zeta=0.032}$, \\
$f=0.394 \mathrm{~Hz}$ & \multicolumn{2}{|c|}{$f=0.505 \mathrm{~Hz}$} & \multicolumn{2}{l|}{$f=0.598 \mathrm{~Hz}$} \\
\hline Signal & Residue & Signal & Residue & Signal & Residue \\
\hline$P_{13-17}$ & 1.000 & $P_{16-18}$ & 1.000 & $P_{13-17}$ & 1.000 \\
$P_{51-45}$ & 0.773 & $P_{14-41}$ & 0.760 & $P_{17-36}$ & 0.698 \\
$P_{51-50}$ & 0.665 & $P_{42-18}$ & 0.727 & $P_{43-17}$ & 0.600 \\
\hline
\end{tabular}

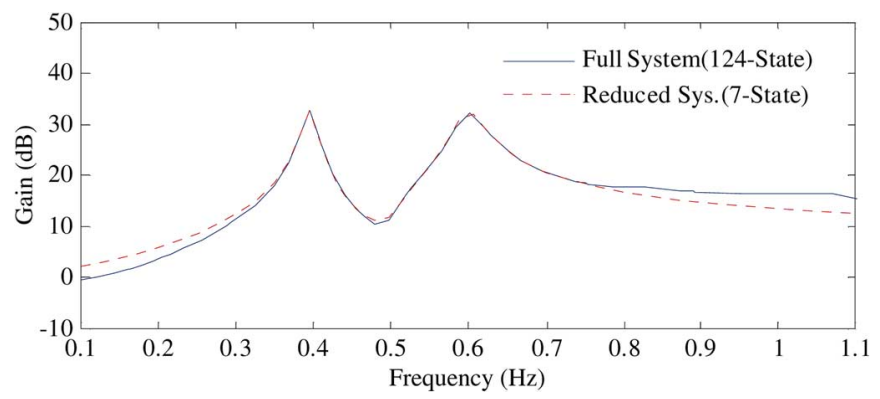

Fig. 6. Frequency response of the full vs. the reduced system.

open loop system response confirmed that the other electromechanical modes including one inter-area mode of the system settled in less than 10 seconds and hence they have been left from the consideration of providing additional damping. The remote feedback signals were chosen based on modal observability analysis [29] for various active line power signals. For the fixed location of actuator, the modal controllability does not change so modal residue is related to modal observability by a scale factor. Table I gives the normalized residues of top 3 active power flows in the 3 inter-area modes.

The signals $P_{13-17}$ (having highest residues for modes 1 and 3) and $P_{16-18}$ (having highest residue for mode 2) have been selected as output signals. With these two signals as output and $k_{c-s s}$, (the control signal of the TCSC) as the input, the open-loop system was linearized to find the state space matrices. System order was reduced (Section II-A) to the lowest possible order such that the reduced system still remained a very good approximation of the full system in the frequency range of $0.2-1.0 \mathrm{~Hz}$; and thus a reduced seventh order system was obtained, as shown in Fig. 6. 


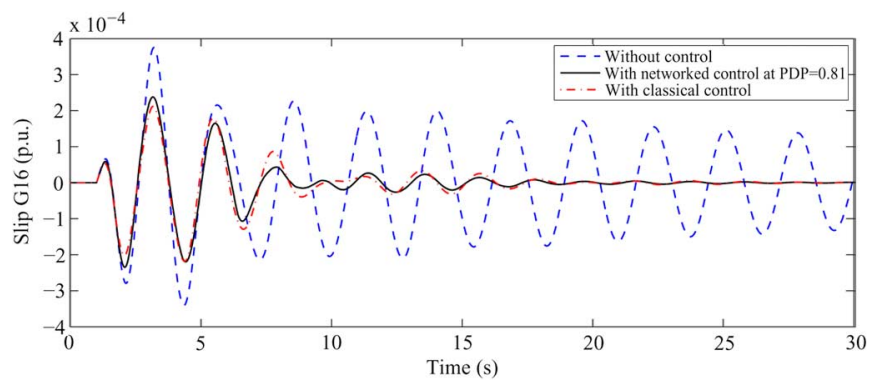

Fig. 7. Rotor-slip response for G16 at operating point 1.

Remark: Deciding the Sampling Period: The controller needs to observe the system in the range of $0.2-1.0 \mathrm{~Hz}$; hence the minimum required sampling frequency is $2.0 \mathrm{~Hz}$ (i.e., a maximum allowed sampling period of $0.5 \mathrm{~s}$ ) according to the NyquistShannon sampling theorem. This upper limit of sampling period is also the threshold requirement for average time delay of the packets, as if the average delay is more than this upper limit, then the packet loss rate will be very high and the network would not support the communication needs of the system. In this paper, a conservative sampling period of $T_{s}=0.1 \mathrm{~s}$ was assumed.

The packet loss in the path of the input and output signals was modeled as a Bernoulli's process. So, $\boldsymbol{\alpha}_{k}=\alpha_{k}^{1}$, while $\boldsymbol{\beta}_{k+1}=$ $\operatorname{diag}\left(\beta_{k+1}^{1}, \beta_{k+1}^{2}\right)$, in (6). The steady state controller gain for the reduced system was found using the results of Section II-D and the modified Kalman filter was modeled using the principle described in Section II-E. Simulation was started and after one second a disturbance was created in the NCSG model by a threephase fault and immediate outage of one of the tie lines between buses 53-54.

The open loop system was a minimum-phase system (i.e., all of its modes were stable); thus it was required to check only the damping response of the system for various packet drop rates. For $\boldsymbol{\alpha}_{k}, \boldsymbol{S}_{p}=\{\emptyset,\{1\}\}$, and $\boldsymbol{T}_{p}(\boldsymbol{r})$ has two modes, $\boldsymbol{T}_{1}(\emptyset)$ and $\boldsymbol{T}_{1}(\{1\})$. For $\boldsymbol{\beta}_{k}, q=2$ and $\boldsymbol{S}_{q}=\{\emptyset,\{1\},\{2\},\{1,2\}\}$, and $\boldsymbol{T}_{q}(\boldsymbol{s})$ has four modes viz. $\boldsymbol{T}_{2}(\emptyset), \boldsymbol{T}_{2}(\{1\}), \boldsymbol{T}_{2}(\{2\})$ and $\boldsymbol{T}_{2}(\{1,2\})$. The corresponding jump state matrices $\mathcal{A}(\boldsymbol{r}, \boldsymbol{s})$ are $\mathcal{A}(\emptyset, \emptyset), \mathcal{A}(\emptyset,\{1\}), \mathcal{A}(\emptyset,\{2\}), \mathcal{A}(\emptyset,\{1,2\}), \mathcal{A}(\{1\}, \emptyset)$, $\boldsymbol{A}(\{1\},\{1\}), \mathcal{A}(\{1\},\{2\})$ and $\mathcal{A}(\{1\},\{1,2\})$, and their probabilities of occurrences are $\left(1-p_{u 1}\right)\left(1-p_{y 1}\right)\left(1-p_{y 2}\right)$, $\left(1-p_{u 1}\right) p_{y 1}\left(1-p_{y 2}\right),\left(1-p_{u 1}\right)\left(1-p_{y 1}\right) p_{y 2},\left(1-p_{u 1}\right) p_{y 1} p_{y 2}$, $p_{u 1}\left(1-p_{y 1}\right)\left(1-p_{y 2}\right), p_{u 1} p_{y 1}\left(1-p_{y 2}\right), p_{u 1}\left(1-p_{y 1}\right) p_{y 2}$, and $p_{u 1} p_{y 1} p_{y 2}$, respectively. Using these parameters, eight pairs of LMIs in (23) were obtained. $\theta$ was taken as 84.3 degrees corresponding to $10 \%$ damping line, as shown in Fig. 4. Assuming same network characteristics for all the network-channels, i.e., $p_{u 1}=p_{y 1}=p_{y 2}=p_{y}$, the feasibility of the LMI's was checked for various values of $p_{y}$ using LMI toolbox in MATLAB. The toolbox returned a minimum feasible value of $p_{y}=0.81$, i.e., the LMIs were feasible for $0.81<p_{y}<1.0$.

Fig. 7 shows the rotor slip response for G16 at the marginal PDP of $p_{y}=0.81$. System response using a classical damping controller [30] (assuming a perfect communication link in its control loop, with infinite sampling rate and zero packet loss) has also been shown for comparison. The details of the classical controller are available in the Appendix C.

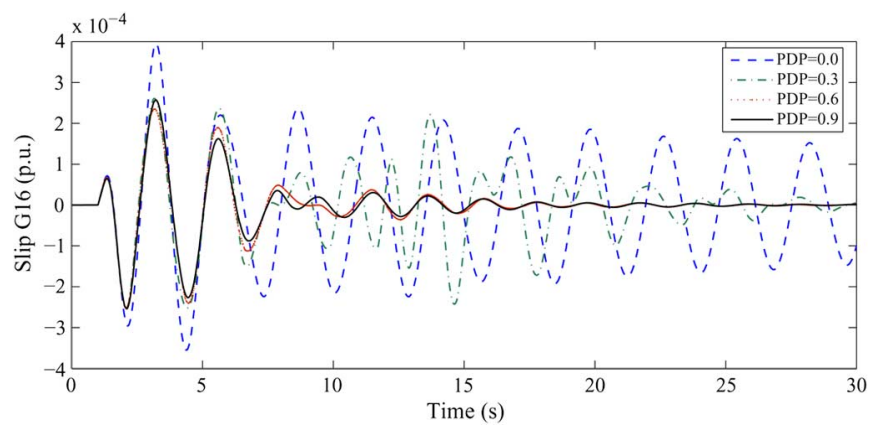

Fig. 8. Rotor-slip response for various packet delivery probabilities (PDPs).

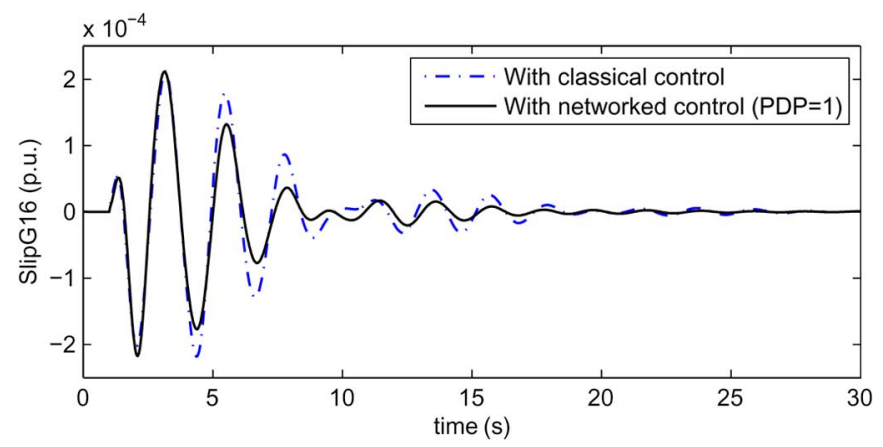

Fig. 9. Classical vs. networked control, with assumption of ideal network conditions.

The rotor-slip response of G16 following the disturbance has also been displayed for four other values of $p_{y}$ as shown in Fig. 8 .

Remark: It should be understood that it is not the sole purpose of Fig. 7 (and subsequent figures) to show that the performance of the networked controller is better than the classical damping controller. Rather, its another important purpose is to show that the performance of the networked control with communication packet-dropout, even with marginal PDP, is comparable to the performance of classical control in which an ideal, lossless and delay-free communication network is assumed. Fig. 9 shows the comparison of the performance of networked control with that of classical control, when in both the cases an ideal communication network is assumed (that is PDP $=1$ ). It can be clearly verified from the figure that the performance of networked control is much better than classical control when ideal network conditions are assumed for both the cases. Also, a metric which is used to assess the control effort required by a control method is the 2-norm of the output from the controller, or $\|\boldsymbol{u}\|_{2}$. The 2-norm for the classical control is 0.32 p.u., while for networked control (with PDP $=1$ ) it is 0.25 p.u. Thus networked control at $100 \%$ packet delivery rate is better than classical control, and it can damp the oscillations in a smaller amount of time, even when the control effort required by networked control is decreased by $22 \%$ as compared to the control effort required by classical control.

2) Operating Condition 2: Total Tieline Flow Between NETS and NYPS $=900 \mathrm{MW}$, No Line Outages: In the second operating condition, the open-loop system becomes unstable after the line-outage, unlike the first operating condition. This is due to the presence of an unstable mode with negative damping ratio in the system. Therefore we may apply either the LMI analysis (Section III-A1) or the stochastic eigen value 


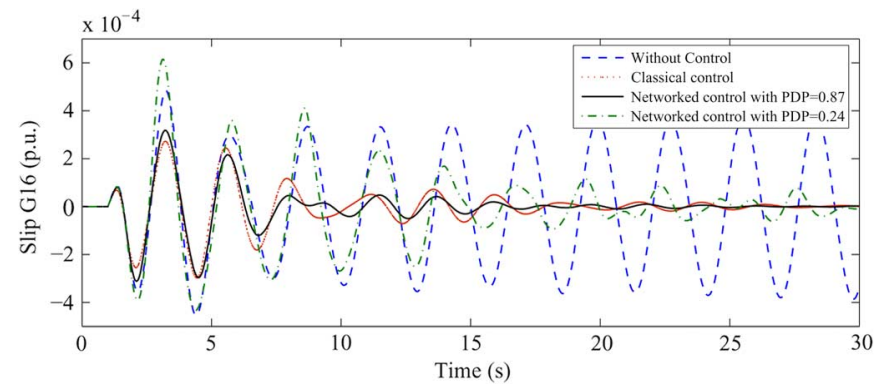

Fig. 10. Rotor-slip response for G16 at operating point 2.

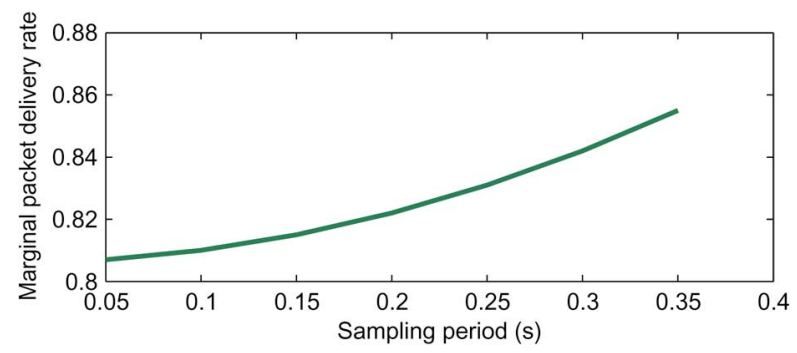

Fig. 11. Marginal delivery probability vs. sampling period.

TABLE II

Marginal Packet Delivery Probability vs. OPerating Point

\begin{tabular}{|c|c|c|c|}
\hline \hline S.No. & Total tieline flow (MW) & Line-outage & Marginal PDP \\
\hline 1. & 700 & no-outage & 0.81 \\
2. & 700 & $60-61$ & 0.83 \\
3. & 700 & $27-53$ & 0.81 \\
4. & 100 & no-outage & 0.79 \\
5. & 900 & no-outage & 0.87 \\
6. & 100 & $27-53$ & 0.79 \\
\hline
\end{tabular}

analysis (Section III-A2) to find the marginal PDP which can ensure closed-loop stability of the NCSG. It was found that the stability of the NCSG under this operating condition was ensured at a marginal PDP of 0.24 , while the adequate damping of the system was ensured at a marginal PDP of 0.87 (using Section III-B). The slip response of G16 has been shown at both of these marginal PDPs in Fig. 10.

It is evident in Figs. 7 and 8, for $T_{s}=0.1 \mathrm{~s}$ and PDPs more than or equal to 0.81 , the inter-area modes of the system are properly damped. Similarly, it may be observed from Fig. 10 that the system in second operating condition is stabilized at the marginal PDP of 0.24 while adequately damped at the marginal PDP of 0.87 . So the results of the LMI analysis stand verified. It is also clear from Fig. 7 and Fig. 10 that the performance of the networked controller is better than the classical damping controller, at realistic packet delivery qualities that can be easily delivered by present day telecom networks.

Next, the effect of the sampling period on the marginal delivery probability for $\mathcal{D}$-stability is being investigated. Fig. 11 shows the plot of the marginal delivery probability versus sampling period. One can easily infer from Fig. 11 that higher sampling period requires increase in $p_{y}$ to guarantee feasibility. This is in line with the expectation that a packet has to be delivered with higher probability with an increase in sampling time.

The robustness of the NCSG has been checked by obtaining the probabilities of marginal packet delivery for various operating conditions as listed in Table II. In Table II, serial number 1
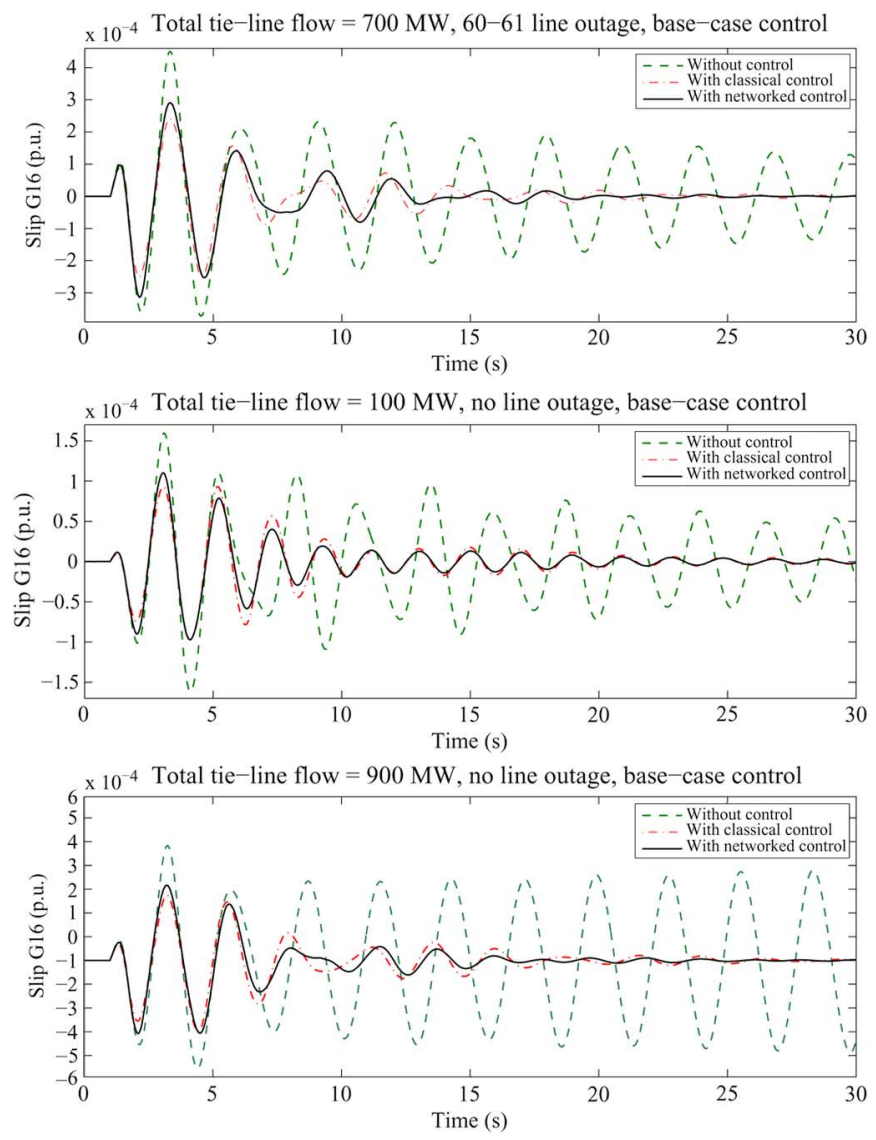

Fig. 12. Rotor-slip response for various operating points at $p_{y}=0.85$.

(S.No.1) was considered the base case of operation. For each operating condition, the control scheme was required to be updated to give a corresponding LQG gain and reduced-order state space matrices for the Kalman filter. The stability performance of the NCSG was also studied with a constant control scheme, i.e., the control scheme obtained for the base case was used for all the operating conditions. Fig. 12 shows the rotor-slip response for 3 operating conditions with such constant control scheme. It is clear from Table II and Fig. 12 that the NCSG is $\mathcal{D}$-stable for various operating points, even with a constant control scheme, at a feasible delivery probability of 0.85 .

\section{CONCLUSIONS}

The effects of introducing a packet-based communication network in the control loops of a power system have been analyzed using the generalized model of a networked controlled smart grid. The random loss in the delivery of the packets has been modeled as a stochastic process. Using the developed linear matrix inequalities, the lower limit on the probability of packet delivery has been computed which guarantees specified damping. It was also found that under varying operating conditions the performance of the NCSG was robust. The contribution of the paper lies in the fact that it not only presents a networked control framework for a power system; but it also presents a formal approach for finding the minimum network requirements in terms of packet delivery quality, so that the specified stability and damping margins can be ensured for any operating condition of a power system. The research findings show that the NCSG framework has a good potential 
to deliver oscillatory stability margin for the smart electricity grid network of the 21 st century.

\section{APPENDIX A}

\section{DETAILS AND DERIVATIONS OF LEMMA 1}

The jump system in (18) is mean square stable iff the matrix $\boldsymbol{M}=\sum_{\boldsymbol{r} \in \boldsymbol{S}_{p}} \sum_{\boldsymbol{s} \in \boldsymbol{S}_{q}}\left(\mathcal{P}_{p}(\boldsymbol{r}) \mathcal{P}_{q}(\boldsymbol{s})[\mathcal{A}(\boldsymbol{r}, \boldsymbol{s}) \otimes \mathcal{A}(\boldsymbol{r}, \boldsymbol{s})]\right)$ is Schur stable (using the stability result of jump linear systems [25]). Rewriting $\mathcal{A}(\boldsymbol{r}, \boldsymbol{s})$ in (21) as $\left[\begin{array}{ll}\boldsymbol{A}_{1} & \boldsymbol{A}_{2} \\ \boldsymbol{A}_{3} & \boldsymbol{A}_{4}\end{array}\right]$, and approximating $B \boldsymbol{T}_{p}(\boldsymbol{r}) \boldsymbol{L}-\boldsymbol{A}^{\prime}=\boldsymbol{B} \boldsymbol{T}_{p}(\boldsymbol{r}) \boldsymbol{L}-\left(\boldsymbol{A}+\boldsymbol{B} \mathbb{E}\left[\boldsymbol{T}_{p}(\boldsymbol{r})\right] \boldsymbol{L}\right) \approx-\boldsymbol{A}$ to simplify the stability analysis, we have $\boldsymbol{A}_{1}=\boldsymbol{A}, \boldsymbol{A}_{2}=\boldsymbol{B} \boldsymbol{T}_{p}(\boldsymbol{r}) \boldsymbol{L}$, $\boldsymbol{A}_{3}=\boldsymbol{\kappa} \boldsymbol{T}_{q}(\boldsymbol{s}) \boldsymbol{C A}, \boldsymbol{A}_{4}=\boldsymbol{A}^{\prime}-\boldsymbol{\kappa} \boldsymbol{T}_{q}(\boldsymbol{s}) \boldsymbol{C A}$, and,

$$
\begin{aligned}
\boldsymbol{M}= & \sum_{\boldsymbol{r} \in \boldsymbol{S}_{p}} \sum_{\boldsymbol{s} \in \boldsymbol{S}_{q}} \boldsymbol{\mathcal { P }}_{p}(\boldsymbol{r}) \mathcal{P}_{q}(\boldsymbol{s})\left[\begin{array}{ll}
\boldsymbol{A}_{1} & \boldsymbol{A}_{2} \\
\boldsymbol{A}_{3} & \boldsymbol{A}_{4}
\end{array}\right] \otimes\left[\begin{array}{lll}
\boldsymbol{A}_{1} & \boldsymbol{A}_{2} \\
\boldsymbol{A}_{3} & \boldsymbol{A}_{4}
\end{array}\right] \\
= & \sum_{\boldsymbol{r} \in \boldsymbol{S}_{p}} \sum_{\boldsymbol{s} \in \boldsymbol{S}_{q}} \boldsymbol{\mathcal { P }}_{p}(\boldsymbol{r}) \mathcal{P}_{q}(\boldsymbol{s}) \\
& \times\left[\begin{array}{llll}
\boldsymbol{A}_{1} \otimes \boldsymbol{A}_{1} & \boldsymbol{A}_{1} \otimes \boldsymbol{A}_{2} & \boldsymbol{A}_{2} \otimes \boldsymbol{A}_{1} & \boldsymbol{A}_{2} \otimes \boldsymbol{A}_{2} \\
\boldsymbol{A}_{1} \otimes \boldsymbol{A}_{3} & \boldsymbol{A}_{1} \otimes \boldsymbol{A}_{4} & \boldsymbol{A}_{2} \otimes \boldsymbol{A}_{3} & \boldsymbol{A}_{2} \otimes \boldsymbol{A}_{4} \\
\boldsymbol{A}_{3} \otimes \boldsymbol{A}_{1} & \boldsymbol{A}_{3} \otimes \boldsymbol{A}_{2} & \boldsymbol{A}_{4} \otimes \boldsymbol{A}_{1} & \boldsymbol{A}_{4} \otimes \boldsymbol{A}_{2} \\
\boldsymbol{A}_{3} \otimes \boldsymbol{A}_{3} & \boldsymbol{A}_{3} \otimes \boldsymbol{A}_{4} & \boldsymbol{A}_{4} \otimes \boldsymbol{A}_{3} & \boldsymbol{A}_{4} \otimes \boldsymbol{A}_{4}
\end{array}\right] \\
= & {\left[\begin{array}{llll}
\boldsymbol{M}_{11} & \boldsymbol{M}_{12} & \boldsymbol{M}_{21} & \boldsymbol{M}_{22} \\
\boldsymbol{M}_{13} & \boldsymbol{M}_{14} & \boldsymbol{M}_{23} & \boldsymbol{M}_{24} \\
\boldsymbol{M}_{31} & \boldsymbol{M}_{32} & \boldsymbol{M}_{41} & \boldsymbol{M}_{42} \\
\boldsymbol{M}_{33} & \boldsymbol{M}_{34} & \boldsymbol{M}_{43} & \boldsymbol{M}_{44}
\end{array}\right]=\boldsymbol{M}\left(\boldsymbol{P}_{\boldsymbol{u}}, \boldsymbol{P}_{\boldsymbol{y}}\right) }
\end{aligned}
$$

The elements of $\boldsymbol{M}\left(\boldsymbol{P}_{\boldsymbol{u}}, \boldsymbol{P}_{\boldsymbol{y}}\right)$ are derived as follows:

$$
\begin{aligned}
\boldsymbol{M}_{11} & =\sum_{\boldsymbol{r} \in \boldsymbol{S}_{p}} \sum_{\boldsymbol{s} \in \boldsymbol{S}_{q}} \mathcal{P}_{p}(\boldsymbol{r}) \mathcal{P}_{q}(\boldsymbol{s})\left[\boldsymbol{A}_{1} \otimes \boldsymbol{A}_{1}\right] \\
& =\left[\sum_{\boldsymbol{r} \in \boldsymbol{S}_{p}} \mathcal{P}_{p}(\boldsymbol{r})\right]\left[\sum_{\boldsymbol{s} \in \boldsymbol{S}_{q}} \mathcal{P}_{q}(\boldsymbol{s})\right]\left[\boldsymbol{A}_{1} \otimes \boldsymbol{A}_{1}\right]=\boldsymbol{A} \otimes \boldsymbol{A}
\end{aligned}
$$

as $\sum_{\boldsymbol{r} \in \boldsymbol{S}_{p}} \boldsymbol{P}_{p}(\boldsymbol{r})=\sum_{\boldsymbol{r} \in \boldsymbol{S}_{p}}\left[\prod_{i \in r} p_{u i} \prod_{i \notin r}\left(1-p_{u i}\right)\right]=1$ and $\sum_{\boldsymbol{s} \in \boldsymbol{S}_{q}} \boldsymbol{\mathcal { P }}_{q}(\boldsymbol{s})=\sum_{\boldsymbol{s} \in \boldsymbol{S}_{q}}\left[\prod_{i \in s} p_{y i} \prod_{i \notin s}\left(1-p_{y i}\right)\right]=1$

$$
\begin{aligned}
\boldsymbol{M}_{12} & =\sum_{\boldsymbol{r} \in \boldsymbol{S}_{p}} \sum_{\boldsymbol{s} \in \boldsymbol{S}_{q}} \mathcal{P}_{p}(\boldsymbol{r}) \mathcal{P}_{q}(\boldsymbol{s})\left[\boldsymbol{A}_{1} \otimes \boldsymbol{A}_{2}\right] \\
& =\left[\sum_{\boldsymbol{s} \in \boldsymbol{S}_{q}} \mathcal{P}_{q}(\boldsymbol{s})\right]\left[\boldsymbol{A} \otimes\left(\boldsymbol{B}\left[\sum_{\boldsymbol{r} \in \boldsymbol{S}_{p}} \mathcal{P}_{p}(\boldsymbol{r}) \boldsymbol{T}_{p}(\boldsymbol{r})\right] \boldsymbol{L}\right)\right] \\
& =\boldsymbol{A} \otimes\left(\boldsymbol{B} \boldsymbol{P}_{u} \boldsymbol{L}\right), \text { as } \sum_{\boldsymbol{r} \in \boldsymbol{S}_{p}} \mathcal{P}_{p}(\boldsymbol{r}) \boldsymbol{T}_{p}(\boldsymbol{r})=\boldsymbol{P}_{u} .
\end{aligned}
$$$$
\boldsymbol{M}_{14}=\sum_{\boldsymbol{r} \in \boldsymbol{S}_{p}} \sum_{\boldsymbol{s} \in \boldsymbol{S}_{q}} \mathcal{P}_{p}(\boldsymbol{r}) \mathcal{P}_{q}(\boldsymbol{s})\left[\boldsymbol{A}_{2} \otimes \boldsymbol{A}_{2}\right]
$$$$
=\sum_{\boldsymbol{s} \in \boldsymbol{S}_{q}} \boldsymbol{\mathcal { P }}_{q}(\boldsymbol{s})
$$$$
\times\left[(\boldsymbol{B} \otimes \boldsymbol{B}) \sum_{\boldsymbol{r} \in \boldsymbol{S}_{p}} \boldsymbol{\mathcal { P }}_{p}(\boldsymbol{r})\left(T_{p}(\boldsymbol{r}) \otimes \boldsymbol{T}_{p}(\boldsymbol{r})\right)(\boldsymbol{L} \otimes \boldsymbol{L})\right]
$$$$
=\left[(\boldsymbol{B} \otimes \boldsymbol{B}) \boldsymbol{F}\left(\boldsymbol{P}_{\mathbf{u}}\right)(\boldsymbol{L} \otimes \boldsymbol{L})\right],
$$$$
\text { as } \sum_{\boldsymbol{r} \in \boldsymbol{S}_{p}} \boldsymbol{\mathcal { P }}_{p}(\boldsymbol{r})\left(T_{p}(\boldsymbol{r}) \otimes \boldsymbol{T}_{p}(\boldsymbol{r})\right)
$$

$=\operatorname{block}-\operatorname{diag}\left(\boldsymbol{F}_{1}, \boldsymbol{F}_{2}, \ldots, \boldsymbol{F}_{p}\right)=\boldsymbol{F}\left(\boldsymbol{P}_{\boldsymbol{u}}\right)$, where,

$F_{i}=p_{u i} \operatorname{diag}\left(p_{u 1}, p_{u 2}, \ldots, p_{u(i-1)}, 1, p_{u(i+1)}, \ldots p_{u p}\right)$
Other terms of $\boldsymbol{M}\left(\boldsymbol{P}_{\boldsymbol{u}}, \boldsymbol{P}_{\boldsymbol{y}}\right)$ are similarly derived as:

$$
\begin{aligned}
\boldsymbol{M}_{13} & =\left(\boldsymbol{B} \boldsymbol{P}_{\boldsymbol{u}} \boldsymbol{L}\right) \otimes \boldsymbol{A}, \boldsymbol{M}_{21}=\boldsymbol{A} \otimes\left(\boldsymbol{\kappa} \boldsymbol{P}_{\boldsymbol{y}} \boldsymbol{C A}\right), \\
\boldsymbol{M}_{22} & =\boldsymbol{A} \otimes\left(\boldsymbol{A}^{\prime}-\boldsymbol{\kappa} \boldsymbol{P}_{\boldsymbol{y}} \boldsymbol{C A}\right), \boldsymbol{M}_{23}=\left(\boldsymbol{B} \boldsymbol{P}_{\boldsymbol{u}} \boldsymbol{L}\right) \otimes\left(\boldsymbol{\kappa} \boldsymbol{P}_{\boldsymbol{y}} \boldsymbol{C A}\right), \\
\boldsymbol{M}_{24} & =\left(\boldsymbol{B} \boldsymbol{P}_{\boldsymbol{u}} \boldsymbol{L}\right) \otimes\left(\boldsymbol{A}^{\prime}-\boldsymbol{\kappa} \boldsymbol{P}_{\boldsymbol{y}} \boldsymbol{C A}\right), \boldsymbol{M}_{31}=\left(\boldsymbol{\kappa} \boldsymbol{P}_{\boldsymbol{y}} \boldsymbol{C A}\right) \otimes \boldsymbol{A}, \\
\boldsymbol{M}_{32} & =\left(\boldsymbol{\kappa} \boldsymbol{P}_{\boldsymbol{y}} \boldsymbol{C A}\right) \otimes\left(\boldsymbol{B} \boldsymbol{P}_{\boldsymbol{u}} \boldsymbol{L}\right), \boldsymbol{M}_{33}=\left(\boldsymbol{A}^{\prime}-\boldsymbol{\kappa} \boldsymbol{P}_{\boldsymbol{y}} \boldsymbol{C A}\right) \otimes \boldsymbol{A}, \\
\boldsymbol{M}_{34} & =\left(\boldsymbol{A}^{\prime}-\boldsymbol{\kappa} \boldsymbol{P}_{\boldsymbol{y}} \boldsymbol{C A}\right) \otimes\left(\boldsymbol{B} \boldsymbol{P}_{\boldsymbol{u}} \boldsymbol{L}\right), \\
\boldsymbol{M}_{41} & =(\boldsymbol{\kappa} \otimes \boldsymbol{\kappa}) \boldsymbol{F}\left(\boldsymbol{P}_{\boldsymbol{y}}\right)(\boldsymbol{C} \otimes \boldsymbol{C})(\boldsymbol{A} \otimes \boldsymbol{A}), \\
\boldsymbol{M}_{42} & =\left(\boldsymbol{\kappa} \boldsymbol{P}_{\boldsymbol{y}} \boldsymbol{C A}\right) \otimes \boldsymbol{A}^{\prime}-\boldsymbol{M}_{41}, \\
\boldsymbol{M}_{43} & =\boldsymbol{A}^{\prime} \otimes\left(\boldsymbol{\kappa} \boldsymbol{P}_{\boldsymbol{y}} \boldsymbol{C A}\right)-\boldsymbol{M}_{41}, \\
\boldsymbol{M}_{44} & =\boldsymbol{A}^{\prime} \otimes \boldsymbol{A}^{\prime}-\boldsymbol{M}_{42}-\boldsymbol{M}_{43}-\boldsymbol{M}_{41}, \\
\boldsymbol{F}\left(\boldsymbol{P}_{\boldsymbol{y}}\right) & =\text { block-diag }\left(\boldsymbol{G}_{1}, \boldsymbol{G}_{2}, \ldots, \boldsymbol{G}_{q}\right), \text { and } \\
\boldsymbol{G}_{i} & =p_{y i} \text { diag }\left(p_{y 1}, p_{y 2}, \ldots, p_{y(i-1)}, 1, p_{y(i+1)}, \ldots p_{y q}\right) .
\end{aligned}
$$

APPENDIX B

PROOF OF LEMMA 2

The damping-region of interest $\mathcal{D}(\theta)$ shown in Fig. 4 is applicable only to the continuous-time representation of a dynamic system; the discrete-time equivalent of this region is a logarithmic spiral and is very difficult to represent using matrices and LMIs. We therefore consider the continuous-time equivalent of $\mathcal{A}(\boldsymbol{r}, \boldsymbol{s})$ which is given by (23) as $\mathcal{A}_{\boldsymbol{c}}(\boldsymbol{r}, \boldsymbol{s})$. Using [27] we know that a dynamic system $\dot{\boldsymbol{x}}=\boldsymbol{A x}$ is $\mathcal{D}$-stable in the region $\mathcal{D}(\theta)$ iff $\boldsymbol{W} \otimes \boldsymbol{A}$ is asymptotically stable. This holds because the eigenvalues of $\boldsymbol{W}$ are $e^{ \pm j((\pi / 2)-\theta)}$. The eigenvalues of the Kronecker product of 2 matrices are the product of the eigenvalues of individual matrices. Hence, the eigenvalues of $\boldsymbol{W} \otimes \boldsymbol{A}$ are two sets of eigenvalues of $\boldsymbol{A}$, one set rotated by an angle $((\pi / 2)-\theta)$ and another one by $-((\pi / 2)-\theta)$. All those eigenvalues of $\boldsymbol{A}$ which lie outside $\mathcal{D}(\theta)$ get rotated into the right half plane in $\boldsymbol{W} \otimes \boldsymbol{A}$, and hence $\boldsymbol{W} \otimes \boldsymbol{A}$ is asymptotically stable iff none of the eigenvalues of $\boldsymbol{A}$ lie outside $\mathcal{D}(\theta)$, i.e., iff $\boldsymbol{A}$ is adequately damped. So, the asymptotic stability of $\boldsymbol{W} \otimes \mathcal{A}_{\boldsymbol{c}}(\boldsymbol{r}, \boldsymbol{s})$ implies $\mathcal{D}$-stability of $\mathcal{A}_{\boldsymbol{c}}(\boldsymbol{r}, \boldsymbol{s})$. As $\mathcal{A}_{\boldsymbol{c}}(\boldsymbol{r}, \boldsymbol{s})$ is a jump-linear mode of the stochastic system in (18) with a modal probability of $\mathcal{P}_{p}(\boldsymbol{r}) \mathcal{P}_{q}(\boldsymbol{s})$, the matrix $\boldsymbol{W} \otimes \mathcal{A}_{\boldsymbol{c}}(\boldsymbol{r}, \boldsymbol{s})$ is also a modal matrix of same probability as $\mathcal{A}_{\boldsymbol{c}}(\boldsymbol{r}, \boldsymbol{s})$; and the mean-square stability of $\boldsymbol{W} \otimes \mathcal{A}_{\boldsymbol{c}}(\boldsymbol{r}, \boldsymbol{s})$ [24] (given by (23)) implies the $\mathcal{D}$-stability of $\mathcal{A}_{\boldsymbol{c}}(\boldsymbol{r}, \boldsymbol{s})$ in a mean square sense, i.e., its electro-mechanical modes are expected to have $\zeta>\cos \theta$.

\section{APPENDIX C}

\section{DetaILS OF THE Classical CONTROLLER}

The transfer function for the classical power oscillation damping (POD) controller used in the text has been evaluated using the theory and results given in [30], and it is as follows:

$$
\begin{aligned}
\Delta k_{c-s s}=P_{13-17}(-0.738)\left[\frac{1+0.138 s}{1+0.725 s}\right]^{2} & \\
& +P_{16-18} 0.925\left[\frac{1+0.182 s}{1+0.949 s}\right]^{2}
\end{aligned}
$$




\section{REFERENCES}

[1] C. Hauser, D. Bakken, and A. Bose, "A failure to communicate: Next generation communication requirements, technologies, architecture for the electric power grid," IEEE Power Energy Mag., vol. 3, pp. 47-55, Mar.-Apr. 2005.

[2] Z. Xie, G. Manimaran, V. Vittal, A. Phadke, and V. Centeno, "An information architecture for future power systems and its reliability analysis," IEEE Trans. Power Syst., vol. 17, pp. 857-863, Aug. 2002.

[3] J. E. Flood, Telecommunication Networks, 2nd ed. London, U.K. IET, 1997.

[4] T. Y. Feng, "A survey of interconnection networks," Computer, vol. 14 , no. 12, pp. 12-27, Dec. 1981.

[5] G. Walsh, H. Ye, and L. Bushnell, "Stability analysis of networked control systems," IEEE Trans. Control Syst. Tech., vol. 10, pp. 438-446, May 2002.

[6] W. Zhang, M. Branicky, and S. Phillips, "Stability of networked control systems," IEEE Control Syst., vol. 21, pp. 84-99, Feb. 2001.

[7] J. Paserba, "Analysis and control of power system oscillations," in CIGRE Special Publication 38.01.07, Technical Brochure no. 111, 1996.

[8] G. Andersson, P. Donalek, R. Farmer, N. Hatziargyriou, I. Kamwa, P. Kundur, N. Martins, J. Paserba, P. Pourbeik, J. Sanchez-Gasca, R. Schulz, A. Stankovic, C. Taylor, and V. Vittal, "Causes of the 2003 major grid blackouts in North America and Europe, recommended means to improve system dynamic performance," IEEE Trans. Power Syst., vol. 20, no. 4, pp. 1922-1928, Nov. 2005.

[9] Y. Tipsuwan and M. Y. Chow, "Control methodologies in networked control systems," Control Eng. Practice, vol. 11, pp. 1099-1111, Oct. 2003.

[10] J. Hespanha, P. Naghshtabrizi, and Y. Xu, "A survey of recent results in networked control systems," Proc. IEEE, vol. 95, pp. 138-162, Jan. 2007.

[11] S. Wang, X. Meng, and T. Chen, "Wide-area control of power systems through delayed network communication," IEEE Trans. Control Syst. Tech., vol. 20, pp. 495-503, Mar. 2012.

[12] H. Li, M. Y. Chow, and Z. Sun, "Eda-based speed control of a networked dc motor system with time delays and packet losses," IEEE Trans. Ind. Electron., vol. 56, pp. 1727-1735, May 2009.

[13] N. R. Chaudhuri, D. Chakraborty, and B. Chaudhuri, "An architecture for facts controllers to deal with bandwidth-constrained communication," IEEE Trans. Power Del., vol. 26, pp. 188-196, Mar. 2011.

[14] K. Padiyar, Power System Dynamics: Stability and Control. Tunbridge Wells, U.K.: Anshan Limited, 2004.

[15] M. A. Pai and P. W. Sauer, Power System Dynamics and Stability. Englewood Cliffs, NJ, USA: Prentice-Hall, 1998.

[16] M. G. Chiang and R. Y. Safonov, "A schur method for balanced model reduction," IEEE Trans. Autom. Control, vol. AC-34, pp. 729-733, 1989.

[17] K. Ogata, Modern Control Engineering, 4th ed. Upper Saddle River, NJ, USA: Prentice-Hall PTR, 2001.

[18] D. P. Bertsekas, Dynamic Programming and Optimal Control, 1st ed. Belmont, MA, USA: Athena Scientific, 1995.

[19] B. Sinopoli, L. Schenato, M. Franceschetti, K. Poolla, and S. Sastry, "Optimal linear lqg control over lossy networks without packet acknowledgment," Asian J. Control, vol. 10, pp. 3-13, Jan. 2008.

[20] X. Yu, J. W. Modestino, and X. Tian, "The accuracy of Markov chain models in predicting packet-loss statistics for a single multiplexer," IEEE Trans. Inf. Theory, vol. 54, no. 1, pp. 489-501, Jan. 2008.

[21] W. D. Koning, "Infinite horizon optimal control of linear discrete time systems with stochastic parameters," Automatica, vol. 18, pp. 443-453, Apr. 1982.

[22] B. Sinopoli, L. Schenato, M. Franceschetti, K. Poolla, M. Jordan, and S. Sastry, "Kalman filtering with intermittent observations," IEEE Trans. Autom. Control, vol. 49, pp. 1453-1464, Sep. 2004.

[23] S. Hu and W. Y. Yan, "Stability of networked control systems subject to input and output packet loss," in Proc. 48th IEEE Conf. Decision Control, Held Jointly With the 28th Chinese Control Conf., Dec. 2009, pp. 6888-6892.
[24] L. E. Ghaoui and M. A. Rami, "Robust state-feedback stabilization of jump linear systems via lmis," Int. J. Robust Nonlinear Control, vol. 6, pp. 1015-1022, Nov. 1996.

[25] Y. Loparo and K. A. Fang, "Stochastic stability of jump linear systems," IEEE Trans. Autom. Control, vol. 47, pp. 1204-1208, Jul. 2002.

[26] M. Chilali and P. Gahinet, " $H_{\infty}$ design with pole placement constraints: An lmi approach," IEEE Trans. Autom. Control, vol. 41, pp. 358-367, Mar. 1996.

[27] D. Arzelier, J. Bernussou, and G. Garcia, "Pole assignment of linear uncertain systems in a sector via a lyapunov-type approach," IEEE Trans. Autom. Control, vol. 38, pp. 1128-1132, Jul. 1993.

[28] B. Pal and B. Chaudhuri, Robust Control in Power Systems. New York: Springer, 2005.

[29] N. Martins and L. T. G. Lima, "Determination of suitable locations for power system stabilizers and static VAR compensators for damping electromechanical oscillations in large scale power systems," IEEE Trans. Power Syst., vol. 5, pp. 1455-1469, Nov. 1990.

[30] M. E. Aboul-Ela, "Damping controller design for power system oscillations using global signals," IEEE Trans. Power Syst., vol. 11, no. 2 pp. 767-773, May 1996.

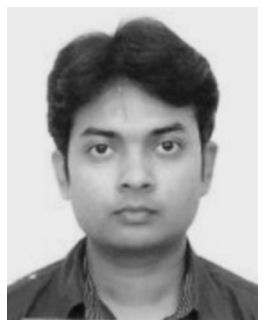

Abhinav Kumar Singh (S'12) received the B.Tech. degree from Indian Institute of Technology, Delhi, India, in 2010 in electrical engineering (power). Currently, he is pursing the Ph.D. degree at the Imperial College London, London, U.K. His current research interests include communication aspects of power systems, dynamic state estimation and power system dynamics.

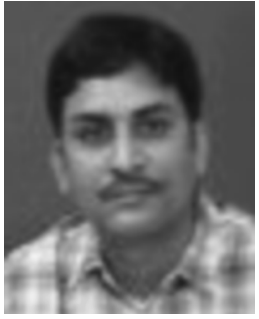

Ravindra Singh (S'07-M'09) received the B.Tech. (with honors) degree from HBTI, Kanpur, India the M.Sc. (Engg.) degree from the Indian Institute of Science, Bangalore, India, and the Ph.D. degree from Imperial College London, U.K., in 2002, 2004, and 2009, respectively, all in electrical engineering. Currently he is working as a Research Scientist at the ABB Corporate Research Center, Raleigh, NC, USA His current research interests include distribution system state estimation and power system dynamics.

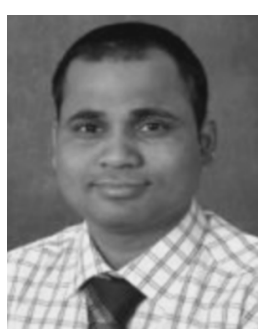

Bikash C. Pal (M'00-SM'02-F'13) received the B.E.E. (with honors) degree from Jadavpur University, Calcutta, India, the M.E. degree from the Indian Institute of Science, Bangalore, India, and the Ph.D. degree from Imperial College London, London, U.K., in 1990, 1992, and 1999, respectively, all in electrical engineering. Currently, he is a Professor in the Department of Electrical and Electronic Engineering, Imperial College London. $\mathrm{He}$ is Editor-in-Chief of IEEE TRANSACTIONS ON SuStainable ENERGY and Fellow of IEEE for his contribution to power system stability and control. His current research interests include state estimation and power system dynamics. 